\title{
Saturation Throughput Performance Analysis of a Medium Transparent MAC Protocol for 60 GHz Radio-Over-Fiber Networks
}

\author{
George Kalfas, Nikos Pleros, Kostos Tsagkaris, Luis Alonso, and Cristos Verikoukis
}

\begin{abstract}
We demonstrate an analytical model for calculating the saturation throughput performance of a medium transparent medium access control (MAC) protocol in $60 \mathrm{GHz}$ radio-over-fiber (RoF) networks. The proposed model incorporates effectively the medium transparent MAC mechanism, assuming a finite number of terminals and ideal channel conditions. It takes into account contention both at the optical and wireless layer, ensuring seamless and dynamic capacity allocation over both transmission media. This model enables extensive saturation throughput performance analysis for the medium transparent MAC and has been applied to $60 \mathrm{GHz}$ RoF network scenarios considering variable numbers of available optical wavelengths, wireless nodes and serving antenna elements and for two different data rate values, namely $155 \mathrm{Mbps}$ and 1 Gbps. Comparison between the model-based throughput results and respective simulation-based outcomes reveals that our model is extremely accurate in predicting the system throughput. Moreover, it confirms that the proposed medium transparent MAC protocol can effectively operate in high-speed $60 \mathrm{GHz}$ RoF LAN environments.
\end{abstract}

Index Terms-Medium access control (MAC) protocol, medium transparent MAC (MT-MAC), performance analysis, radio over fiber (RoF), $60 \mathrm{GHz}$ local access network (LAN), $60 \mathrm{GHz}$ wireless.

\section{INTRODUCTION}

$\mathbf{R}$ ADIO-OVER-FIBER (RoF) technologies have drawn attention as a highly effective paradigm for bridging the ultrafast optical buses with the increasingly utilized wireless connectivity systems [1]. RoF architectures present a cost effective way for extended range passive optical-wireless networks, consolidating all required network intelligence in a single central

Manuscript received June 08, 2011; revised September 06, 2011, October 04, 2011; accepted October 04, 2011. Date of publication October 14, 2011; date of current version December 14, 2011. This work was supported in part by the Research Project GreenNet (264759) and by the Greek General Secretariat for Research and Technology through Project WisePON.

G. Kalfas is with the Technical University of Catalonia, 08034 Barcelona, Spain, and also with the Informatics and Telematics Institute, Center for Research and Technology Hellas, GR-54639 Thessaloniki, Greece (e-mail: george. kalfas@estudiant.upc.edu).

N. Pleros is with the Department of Informatics, Aristotle University of Thessaloniki, GR-54124 Thessaloniki, Greece (e-mail: npleros@csd.auth.gr).

K. Tsagkaris is with the Department of Digital Systems, University of Piraeus, GR-18352 Piraeus, Greece (e-mail: ktsagk@unipi.gr).

L. Alonso is with the Technical University of Catalonia, 08034 Barcelona, Spain (e-mail: luisg@tsc.upc.edu).

C. Verikoukis is with the Telecommunications Technological Centre of Catalonia, 08860 Castelldefels, Spain (e-mail: cveri@cttc.es).

Color versions of one or more of the figures in this paper are available online at http://ieeexplore.ieee.org.

Digital Object Identifier 10.1109/JLT.2011.2172392 unit, well-known as the central office (CO), and relaxing in this way the complexity, functionality, and cost requirements at the terminal equipment. As a result, the deployment of an array of inexpensive remote antenna units (RAUs) can lead to vast coverage wireless networks that optimally combine the mobility and ubiquity advantages of a wireless link with the high speed and long-distance service delivery credentials of fiber-based infrastructure [2].

The majority of related research has focused so far on exploiting the underlying fiber infrastructure as a passive distribution network used solely for the purpose of long-distance wireless service delivery. Within this frame, the research emphasis has been on physical layer technologies and architectures for high-capacity RoF network deployments [3]-[6], whereas only a limited number of efforts have attempted the functional convergence of the optical and wireless network parts through the realization of dynamic capacity allocation and user mobility concepts [7]-[10]. On the same line, only a small portion of the research carried out so far has been dedicated to medium access control (MAC) layer issues, the main interest being in adapting existing MAC protocol standards, like the $802.11 \mathrm{~g}$, to the higher delay metrics of long-distance RoF implementations [11]-[16]. However, this roadmap does not promote the functional interfacing of the currently distinct operative portfolios of the optical and wireless parts, impeding in this way a seamless and powerful optical and wireless network convergence with high-level agility and flexibility potential.

RoF advantages become increasingly important as the unprecedented escalation in wireless bandwidth demand drives the need for employment of wireless frequencies capable of delivering very high data rates. A noteworthy example is the license exempt $60 \mathrm{GHz}$ band which has by now been adopted by the industry as the prevailing candidate region for broadband wireless data transfer. The $60 \mathrm{GHz}$ spectral band has already been enforced in a significant number of emerging standards such as the 802.11 ad [17], the 802.15.3c [18]-[20], the WirelessHD [21] and the WiGig [22] protocols. However, the high air propagation losses of this millimeter-wave radio band constitute a significant constraint in terms of extended range coverage, so that $60 \mathrm{GHz}$ connectivity is inevitably bound to personal area networking (PAN) applications. $60 \mathrm{GHz}$ communication over RoF physical layer implementations holds the credentials for overcoming this range handicap by employing the fiber-based network part for high-distance signal delivery. Moreover, $60 \mathrm{GHz}$ RoF physical layer implementations have reached due maturity for enabling seamless and efficient distribution of multiple 60 
$\mathrm{GHz}$ wireless signals over wavelength division multiplexing infrastructures [3]-[7]. However, the functional convergence of the $60 \mathrm{GHz}$ wireless layer with an underlying RoF platform has not been efficiently addressed so far, lacking an effective mechanism for arbitrating the capacity and the service requests arising by the entire number of antennae elements that are connected to a single CO. Such a scenario would yield to medium transparent traffic control and would certainly allow for the transformation of multiple PANs formed around every individual 60 $\mathrm{GHz}$ RAU into an extended LAN, enabling full reconfigurability of the bandwidth allocated at each terminal. The first and so far solely available medium transparent MAC (MT-MAC) protocol capable to yield successful extended LAN functionalities in $60 \mathrm{GHz}$ broadband wireless over fiber connections has been only recently presented [23], highlighting the advantages of such an archetype and indicating at the same time the potential of these solutions to penetrate $60 \mathrm{GHz}$ LAN evolution.

In this paper, we demonstrate for the first time to our knowledge an analytical model followed by a detailed saturation throughput performance analysis for the MT-MAC protocol described in [23], assuming ideal channel conditions. The introduced model relies on a 2-D Markov chain approach for calculating the end-user transmission probabilities, taking into account contention for both the optical and the wireless layer resources. An analytic formula for throughput computation is derived and the respective results for different optical resource availability factors and for data rates up to $1 \mathrm{Gbps}$ are found to be in close agreement with simulation-based findings, confirming the validity of the MT-MAC model. Our analysis reveals that the proposed MT-MAC is capable of resolving contention within a limited time frame, concluding with the optimized duration of consecutive data transmitting frames for maximizing network throughput. This first successful MT-MAC modeling approach indicates that the functional interfacing of the optical and the wireless connection links can yield new and efficient capacity utilization concepts in $60 \mathrm{GHz}$ RoF networks.

This paper is outlined as follows. Section II briefly reviews the MT-MAC protocol characteristics. Section III includes the analytical throughput analysis describing the MT-MAC mathematical model, while Section IV presents the obtained analytical results comparing them with respective simulation-based outcomes. Sections V and VI discuss and conclude the paper, respectively.

\section{MT-MAC PROTOCOL}

This section describes the principal characteristics of the protocol and the crucial traffic format parameters that directly affect the throughput calculation. A more detailed and complete description of the MT-MAC protocol mechanism and its foundational $60 \mathrm{GHz}$ RoF physical layer architecture is provided in [23].

According to the protocol specifications, all traffic exchange is considered to take place over both the optical and wireless media with the fiber-based network delivering data from the single CO to multiple RAUs, whereas the $60 \mathrm{GHz}$ wireless counterpart is responsible for the link establishment between each RAU and its associated wireless end users. All downlink and uplink data channels are assumed to be generated at the $\mathrm{CO}$ as wavelength pairs $\left\{\lambda_{x}, \lambda_{x}^{\prime}\right\}$ : one wavelength carries the 60 $\mathrm{GHz}$ single-side band (SSB) subcarrier-modulated downlink information, while its respective uplink wavelength is transmitted as a continuous wave into the network and gets $60 \mathrm{GHz}$ SSB subcarrier-modulated with the uplink data at the RAU. A distinct wavelength pair $\left\{\lambda_{c}, \lambda_{c}^{\prime}\right\}$ is specially designated as the common control channel (CC) for all RAU elements, comprising again a dedicated downlink control wavelength and its uplink control analog. Optical-to-RF and RF-to-optical conversion for downlink and uplink transmission, respectively, is realized at the RAUs devices. Discrimination between the data and control signals in the wireless domain is ensured by utilizing a frequency division multiplexing scheme, with the $\mathrm{CC}$ carrier frequency lying outside the bandwidth occupied by the $60 \mathrm{GHz}$ data signals.

The overall media capacity is directly negotiated between the wireless nodes and the $\mathrm{CO}$ without any decisive intervention of the RAUs. This turns the network into a totally transparent entity for the end users who are fully unaware of any infrastructural details, gaining in this way the impression of an uninterrupted horizontal link that leads directly to the CO. The latter performs as the central hub and enables the establishment of an extended reach LAN network between all end users served by it, even when they are located at different RAU cells and no line-of-sight conditions apply between them. The resource allocation process is divided into two parallel contention periods: the first contention period is carried out in the optical domain and decides on the per RAU optical capacity allocation, whereas the second contention period is responsible for the distribution of the wireless bandwidth between nodes located in the same RAU.

During the first contention period, the $\mathrm{CO}$ emits a short optical beacon pulse in the $\mathrm{CC}$ that is in turn broadcasted into the air through every RAU. When in-range nodes containing pending uplink data detect the pulse, they respond immediately by emitting a short pulse of the same duration in order to notify of their existence. These pulses are received by the respective RAU and modulate its allocated time slots in the uplink wavelength channel, which subsequently propagates toward the CO. This procedure is illustrated for an example of four different RAUs served by the $\mathrm{CO}$ in Fig. 1, revealing also the need for having clearly time-discriminated RAU response pulses in order to avoid overlapping between their replies. This can be easily achieved even with pulse durations higher than $10 \mathrm{~ns}$, taking into account the differential fiber delays between the RAUs that are usually located at different distances from the $\mathrm{CO}$.

The synchronized reception of the $\mathrm{CC}$ response pulses informs the $\mathrm{CO}$ about the RAUs containing capacity requesting nodes. Following the reception of the uplink wavelength, the $\mathrm{CO}$ assigns a data transmission wavelength pair to each RAU, ergo ending the first contention period. In high load conditions, where the number of RAUs containing active nodes exceeds the number of available data wavelength pairs, the $\mathrm{CO}$ assigns the wavelengths in a round-robin fashion distributing the available bandwidth amongst all RAUs in a fair, starvation free manner.

The second contention period takes place entirely in the wireless $60 \mathrm{GHz}$ channel, where traffic exchange is logically divided 


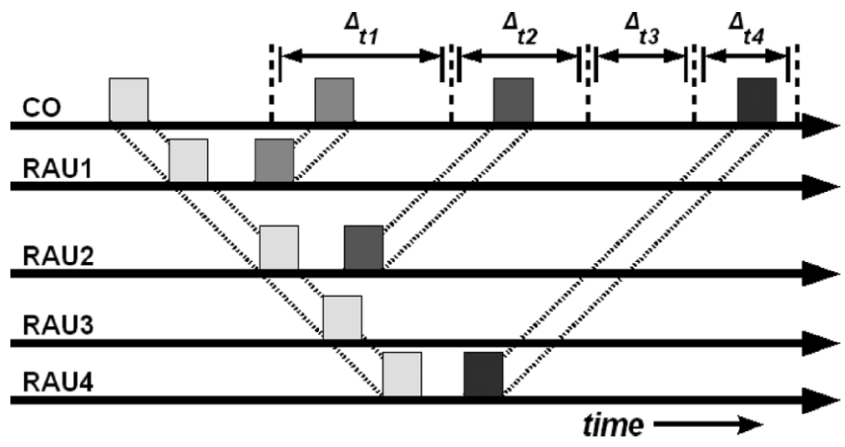

Fig. 1. Traffic requests collection procedure during the first contention period in the case of active nodes located within RAUs 1, 2, and 4.

into superframes (SFs). An SF contains frames with each one being either a resource requesting frame (RRF) or a data frame (DF), as shown in Fig. 2. Both types of frames are of equal size in the air and are broadcasted in non-interchangeable, continuous sets, with the set of RRFs always preceding the group of DFs. Each RRF aims at the identification of the active nodes residing within the RAU cell so as to increase bandwidth utilization by allowing only active nodes' participation in the subsequent DFs. After a number of RRF frames, once the contending end users have been identified and, therefore, resolved, DFs are sequentially transmitted carrying out the actual data exchange on the basis of a polling-based scheme. RRFs' structure is further divided into a fixed number of $m$ slots, with each slot comprising POLL, ID, and ACK packets, whereas DFs contain DATA packets instead of ID packets. The number of slots $m$ remains immutable and is predefined by the $\mathrm{CO}$ based on the projected mean number of active nodes per RAU for the final purpose of minimizing, to the extent possible, the number of RRFs utilized in the second contention period. At the beginning of each RRF, all the active nodes choose an integer value $y$ in the interval $[0, m)$, where $y$ follows a uniform distribution and corresponds to the number of POLL packets that have to be received by the terminal before responding with an ID packet. During an RRF slot, the CO transmits a general POLL packet with no receiving node specified in its body. Upon correct ID packet reception, the $\mathrm{CO}$ responds with an ACK packet, notifying the corresponding node that it has been correctly identified. This node will not participate in a subsequent RRF (if any) within the current SF. Should, however, two nodes choose the same $y$ value, they will transmit an ID packet during the same slot. The collision will render both ID packets unreadable and the $\mathrm{CO}$ will not respond with an ACK, forcing the nodes to participate in the next RRF after choosing a new $y$ value. The CO continues transmitting RRFs until zero collisions occur and, therefore, all nodes are successfully identified, ending in this way the second contention period. Having full knowledge of the nodes that are active within a RAU, the CO initiates the DATA_TX period where the main exchange of data occurs in the form of a series of DFs transmissions until the end of the $\mathrm{SF}$ is reached. In parallel to the data exchange, the CO periodically reruns the first contention period process in the $\mathrm{CC}$ so as to update the list of RAUs requesting traffic. If no known active nodes exist within a newly wavelength allocated RAU, the second contention period process is repeated.

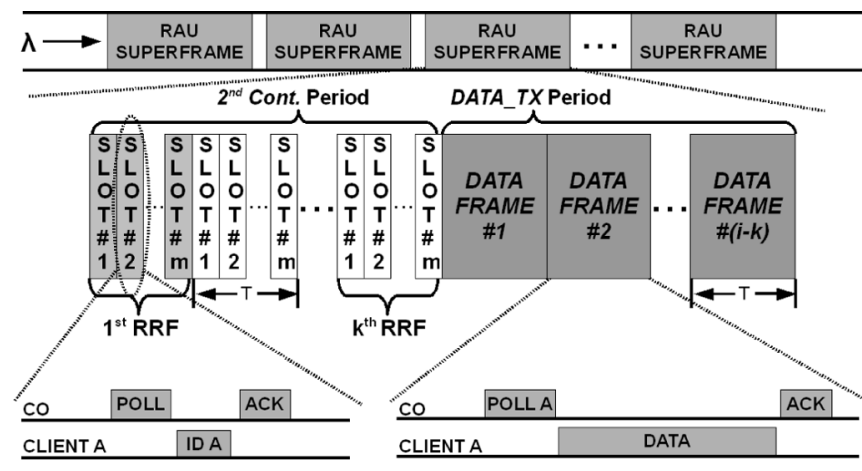

Fig. 2. SF, RF, and DF structure. Nonshaded RRFs are optional.

The CO performs all packet synchronization and scheduling tasks, which are mainly influenced by two architectural characteristics: the optical propagation delay $\delta_{\text {FIBER }}$ induced by fiber's length and the wireless transmission delay $D_{T}$ which is inversely proportional to system's bit rate. In cases where $\delta_{\text {FIBER }}$ and $D_{\boldsymbol{T}}$ are of the same magnitude order, be it due to great distance between the $\mathrm{CO}$ and the RAU modules or due to very high wireless bit rates, packets in the air get sent a lot faster than they travel through the fiber, resulting to idle time periods in the wireless domain. To nullify the latter phenomenon, the $\mathrm{CO}$ performs some minor changes regarding the protocol's operation to the extent required by the corresponding $\delta_{\text {FIBER }} / D_{T}$ ratio. Specifically, to avoid unnecessary delays in the second contention period, the $\mathrm{CO}$ may choose to transmit all POLL packets sequentially and acknowledge the incoming IDs with an aggregated ACK at the end of the RRF. POLL packets in this case are appropriately separated so as not to collide in the air with incoming ID packets, but simultaneously transmitted over the fiber so as to avoid all the intermediate optical round-trip delays, taking advantage of the different uplink and downlink wavelengths. The same principle also applies in the DATA_TX period where the CO may choose to transmit POLL packets sequentially and, following a timely deferred correct DATA reception, piggyback the corresponding ACK to a subsequent POLL packet. This approach, enabled by the centralized nature of the protocol, effectively nullifies all the intermediate fiber round-trip delays among continuous DFs or among the RRF's slots, taking greater advantage of medium's duality and resulting in higher throughput values.

\section{ThroughPUT ANALYSIS}

We declare $S$ to be the normalized system throughput, i.e., the ratio depicting the amount of time that the system transmits payload bits to the total amount of communication time. Considering the protocol rules, the aforementioned translates directly to the time portion that the system exhibits engaged SF activity, and more specifically to the time portion that the system is located in the DATA_TX period of the latter.

Therefore, $S$ can be defined as $S=$ $\left(\Pi_{\mathrm{TX}} T_{\mathrm{DF}}\right) /\left(\Pi_{\mathrm{CONT}} T_{\mathrm{RRF}}+\Pi_{\mathrm{TX}} T_{\mathrm{DF}}\right) \cdot\left(\Pi_{\mathrm{TX}}+\Pi_{\mathrm{CONT}}\right) \cdot$ $\rho_{\mathrm{DF}}$, where $\Pi_{\mathrm{TX}}$ is the steady-state probability (SSP) of the system being in the DATA_TX mode, $\Pi_{\mathrm{CONT}}$ is the SSP of the system being in the second contention period, $T_{D F}$ and $T_{R R F}$ are the durations of the $D F$ and 


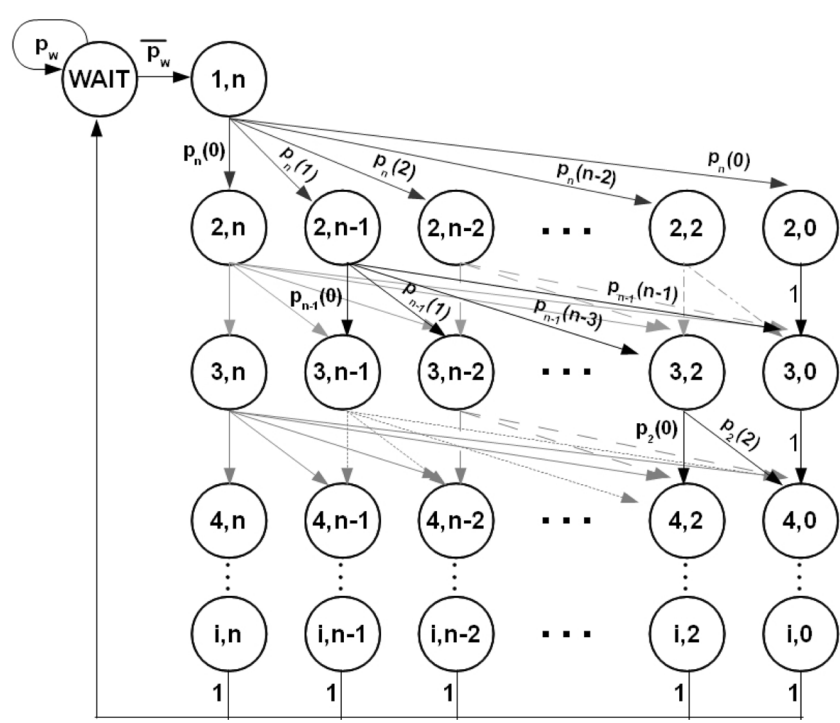

Fig. 3. Markov model from the perspective of a single remote access unit.

the RRF, respectively, and $\rho_{\mathrm{DF}}$ is the payload information percentage contained in a DF. The latter is typically defined as $\rho_{\mathrm{DF}}=L_{\mathrm{DATA}} /\left(L_{\mathrm{POLL}}+L_{\mathrm{DATA}}+L_{\mathrm{ACK}}\right)$ where $L_{\mathrm{DATA}}, L_{\mathrm{POLL}}$, and $L_{\mathrm{ACK}}$ are the size in bytes of the DATA, POLL, and ACK packets, respectively.

To calculate $\Pi_{\mathrm{TX}}$ and $\Pi_{\mathrm{CONT}}$, we have deployed a 2-D Markov chain model, depicted in Fig. 3, which demonstrates scheme's operation from the scope of a single RAU. The aforementioned model operates under the assumption of ideal channel conditions. In addition, we consider that each RAU contains a constant and finite number of $n$ stations in its radius. Traffic generation follows the saturation model, meaning that each of the $n$ terminals always possesses a packet ready for transmission immediately after transmitting the previous one. Finally, no hidden or exposed terminals phenomena are taken into account, since they are negated by our protocol's centralized topology.

In the following analysis, we consider a fixed number of RAUs $R$, with each RAU servicing an identical and constant number of wireless nodes. Moreover, we define the total number of available downlink and uplink data wavelength pairs as $\lambda$. Model's nomenclature follows the $S_{i, \boldsymbol{k}}$ symbolism, where $i$ stands for the $i$ th frame of the current SF and $k$ is the number of nodes that are yet to be resolved through means described in the second contention period. It should be noted here that the first state of the Markov chain model, referred as WAIT, represents the optical waiting period, i.e., the state where the RAU does not have an assigned pair of wavelengths yet and is waiting for optical capacity assignment. Each SF contains a number of frames between $\left(i_{\mathrm{MIN}}, i_{\mathrm{MAX}}\right)$, the values of which are immutable and known to the network. These bounds denote the minimum and maximum time duration of the non-interrupted wavelength assignment to every RAU, respectively. More specifically, the $i_{\mathrm{MIN}}$ and $i_{\mathrm{MAX}}$ values reflect the logical restriction that each consecutive wavelength assignment period must be long enough to allow for adequate data transmission without exceeding, however, a certain upper limit, so as to prevent unaffordable delays and inequalities amongst the RAUs.

Contention in the optical domain is resolved by means of a simple round-robin algorithm using a step of one RAU at the end of each time quantum, i.e., each RAU holds the same wavelength pair for the amount of time equal to the $\lambda / R$ percentage of the total time. In real non-saturated mode of operation, a phase shift in wavelength re-assignment is not performed unless there are outstanding claims for allocating optical capacity to other non-serviced RAUs [23]. However, in network saturation conditions, as considered in our analysis, the $\mathrm{CO}$ acquires a constant demand for optical capacity from all RAUs, implying the exercise of the round-robin algorithm in a strictly time-sharing fashion.

The Markov chain model diagram can be logically divided into two areas. The first area is comprised of the state WAIT, whereas the second area is comprised of all the rest states. State WAIT, being representative of the waiting period caused by the assignment/deassignment of the optical wavelength, effectively controls the length of time that the current RAU lies in idle state. This signifies that its respective SSP is

$$
\Pi_{\mathrm{WAIT}}=1-\lambda / R
$$

and by considering the normalizing condition $\sum \Pi=1$, the sum of the rest SSPs is

$$
\begin{aligned}
& \sum_{i} \sum_{k} \Pi_{i, k}+\Pi_{1, n}=\lambda / R \\
& \qquad \text { for } i \in[2, n], k \in[0, n] \backslash\{1\}
\end{aligned}
$$

where $\Pi_{1, n}$ is the SSP of the $S_{1, n}$ state and accordingly $\Pi_{i, k}$ represents the SSP of the $S_{i, \boldsymbol{k}}$ state. Once being in the WAIT state, the only possible actions are: to remain stationary with probability $p_{\boldsymbol{w}}$ while awaiting for wavelength assignment, or to enter the $S_{\mathbf{1}, \boldsymbol{n}}$ state with probability $\overline{p_{w}}$ once a wavelength has been assigned. Following this, the wireless nodes served by this RAU enter the second contention period according to the rules described in Section II. All the wireless activity is depicted in the second logical area of the Markov model, which can be further divided into distinct rows and columns: each individual row corresponds to a single frame in the SF, while each column represents the number of nodes that are yet to be resolved in the second contention period. As such, the far-left column signifies the maximum number of unresolved nodes $n$ and the far-right column represents the situation where all nodes have been resolved, i.e., the number of unresolved nodes has reached zero. It should be noted that $S_{i, 1}$ type of states are not present in the Markov state diagram, given that node collision can only occur when at least two nodes are available for picking the same slot number in the random selection process.

Every state in this second logical area of the Markov model is accessible from all the states of its preceding frame (or previous row) that lie exactly above or on its left. This carries the physical meaning that the number of unresolved nodes can either remain the same or decrease when moving to the next frame. The transition probability from state $S_{\boldsymbol{i}, \boldsymbol{k}}$ to state $S_{\boldsymbol{i}+\mathbf{1}, \boldsymbol{k}-\boldsymbol{x}}$ equals to the probability of having $x$ out of a current total of $k$ unresolved 
nodes making a unique number choice and, consequently, getting resolved. This probability is provided by the relation [24]

$$
p_{k}(x)=\frac{(-1)^{x} m ! k !}{x !} \cdot \sum_{a=x}^{k} \frac{(-1)^{a}(m-a)^{k-a}}{(a-x) !(m-a) !(k-a) !}
$$

where $m$ is the number of slots the nodes are choosing from, i.e., the slots contained in a single RRF, and $k \in[2, n], x \in$ $[0, k] \backslash\{k-1\}$.

A complete summary of all the non-null one-step transition probabilities is provided as follows:

$$
\left\{\begin{array}{l}
p\{1, n \mid 0, n\}=\overline{p_{W}} \\
p\{i+1, k-x \mid i, k\}=p_{k}(x) \\
p\{i+1,0 \mid i, 0\}=1
\end{array}\right.
$$

for $i \in\left[1, i_{\mathrm{MAX}}\right), k \in[0, n] \backslash\{1\}, x \in[0, k] \backslash\{k-1\}$.

The " $0, n$ " notation in the first part of (4) represents the WAIT state. The third part of (4) denotes that, once reaching a state where all nodes have been resolved, the second contention period is over and the $\mathrm{CO}$ initiates the transmission of sequential DFs until the end of the SF. To this end, the states of the form $S_{i, 0}$ correspond to effective packet transmission, so that the aggregate probability $\Pi_{\mathrm{TX}}$ of the system residing in DATA_TX is

$$
\Pi_{\mathrm{TX}}=\sum_{i=2}^{i_{\mathrm{MAX}}} \Pi_{i, 0} .
$$

Accordingly, all the rest states of the form $S_{i, \boldsymbol{k}}$ for $k \geq 2$ correspond to the second contention period and therefore

$$
\Pi_{\mathrm{CONT}}=\sum_{i=1}^{i_{\mathrm{MAX}}} \sum_{k=2}^{n} \Pi_{i, k} .
$$

All $\Pi_{i, k}$ probabilities including $\Pi_{i, 0}$ can be calculated by exploiting the single-step transitions from all possible states in the preceding $i-1$ row. Every SSP is derived by the sum of all the states that reside in the previous frame and on the left, multiplied by the corresponding transition probabilities. To this end, the SSPs for all $\mathrm{S}_{\boldsymbol{i} . \boldsymbol{k}}$ states can be expressed as

$$
\Pi_{i, k}=\sum_{a=k}^{n}\left(\Pi_{i-1, a} \cdot p_{a}(a-k)\right)
$$

for $i \in\left[2, i_{\mathrm{MAX}}\right], k \in[0, n] \backslash\{1\}$.

For $i=1$, the respective expression describing the transition from the WAIT state to the $S_{1, n}$ is given by

$$
\Pi_{1, n}=\Pi_{\mathrm{WAIT}} \cdot \overline{p_{w}} \text {. }
$$

By utilizing the fact that each of the $i$ frames, independently of its type, is of equal duration we derive that

$$
\begin{aligned}
\Pi_{1, n} & =\sum_{k} \Pi_{2, k}=\sum_{k} \Pi_{3, k}=\cdots \\
& =\sum_{k} \Pi_{i_{\mathrm{MAX}}, k}=\lambda /(i R) .
\end{aligned}
$$

Using (1), (7), and (8), $\overline{p_{W}}$ is found to be

$$
\overline{p_{W}}=\lambda /(i \cdot(R-\lambda)) .
$$

By utilizing (2)-(4), (8), and (10), (7) can be recursively solved yielding all required $\Pi_{i, 0}$ values, which can be then used in (5) to enable the calculation of $\Pi_{\mathrm{TX}}$. Finally, in order to calculate $S$, we also need to specify the $T_{\mathrm{RRF}}$ and $T_{\mathrm{DF}}$ values. According to the protocol rules, $T_{\mathrm{RRF}}$ is defined as

$$
\begin{aligned}
T_{\mathrm{RRF}}=m\left(3 \delta_{\mathrm{FIBER}}+3 \delta_{\mathrm{AIR}}+\right. & D_{\mathrm{T} \_\mathrm{POLL}} \\
& \left.+D_{\mathrm{T} \_\mathrm{ID}}+D_{\mathrm{T} \_\mathrm{ACK}}\right)
\end{aligned}
$$

where $\delta_{\mathrm{AIR}}$ is the propagation delay in the wireless medium and $D_{\mathrm{T} \_ \text {POLL }}, D_{\mathrm{T} \_\mathrm{D}}$, and $D_{\mathrm{T} \_\mathrm{ACK}}$ are the transmission delays of the POLL, ID, and ACK packets, respectively. Correspondingly, $T_{\mathrm{DF}}$ is defined as

$$
\begin{array}{r}
T_{\mathrm{DF}}=3 \delta_{\mathrm{FIBER}}+3 \delta_{\mathrm{AIR}}+D_{\mathrm{T} \_\mathrm{POLL}}+D_{\mathrm{T} \_\mathrm{DATA}} \\
+D_{\mathrm{T} \_\mathrm{ACK}}
\end{array}
$$

where $D_{\mathrm{T} \_ \text {DATA }}$ is the transmission delay of the DATA packet. Equations (11) and (12) correspond to the time duration of the RRF and DF frames, respectively, in the general scenario where no packet rescheduling is taking place. In the latter case though, (11) and (12) become (13) and (14), respectively, where $\Pi_{\mathrm{TX}}$. $i_{\text {MAX }}$ corresponds to the number of DFs in the SF. Notably, if the appropriate values are chosen so that $T_{\mathrm{RRF}}=T_{\mathrm{DF}}$, then $S=\Pi_{\mathrm{TX}} \cdot \rho_{\mathrm{DF}}$.

\section{Performance Results}

To evaluate the validity of our proposed model, we have produced and compared both analytical and simulation results using our custom made event-driven simulation tool written in Java. The employed network configuration comprises 10 RAUs in a bus topology, with each RAU servicing five end users under saturation load conditions, as it is schematically depicted in Fig. 4. Table I summarizes the full specification parameters of the simulation run and of the respective analytical model where applicable.

$$
\begin{aligned}
& T_{\mathrm{RRF}}=3 \delta_{\mathrm{FIBER}}+m\left(3 \delta_{\mathrm{AIR}}+D_{\mathrm{T} \_\mathrm{POLL}}+D_{\mathrm{T} \_\mathrm{ID}}+D_{\mathrm{T} \_\mathrm{ACK}}\right) \\
& \text { and } \\
& T_{\mathrm{DF}}=\frac{2 \delta_{\mathrm{FIBER}}+3 \delta_{\mathrm{AIR}}+\Pi_{\mathrm{TX}} \cdot i_{\mathrm{MAX}}\left(D_{\mathrm{T} \_\mathrm{POLL}}+D_{\mathrm{T} \_\mathrm{DATA}}+D_{\mathrm{T} \_\mathrm{ACK}}\right)}{\Pi_{\mathrm{TX}} \cdot i_{\mathrm{MAX}}}
\end{aligned}
$$




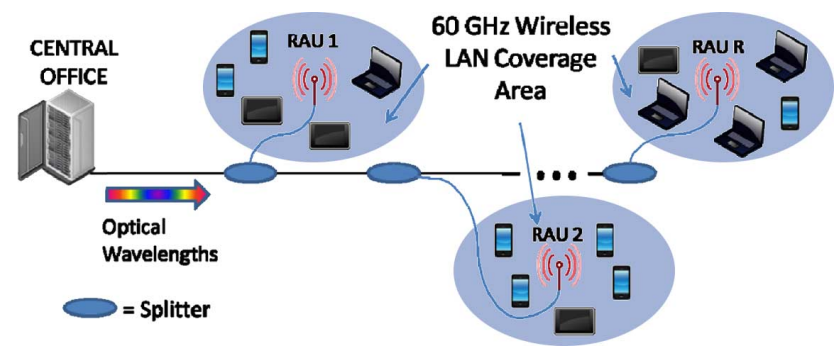

Fig. 4. Performance evaluation configuration.

TABLE I

Simulation Parameters With SATURATion TrafFic Model

\begin{tabular}{|l|c|l|c|}
\hline Fiber Prop. Delay & $1 \mu$ s per $200 \mathrm{~m}$ & Air Prop. Delay & $0.16 \mu \mathrm{s}$ \\
\hline Slots in $R R F$ & 10 & ACK Size & 8 bytes \\
\hline DATA Size & 1288 bytes & ID, POLL Size & 64 bytes \\
\hline Data Bitrate & $155 \mathrm{Mbps}$ & $1 \mathrm{Gbps}$ & $1 \mathrm{Gbps}$ \\
\hline Frames in $S F$ & \multicolumn{2}{|c|}{500} & 3200 \\
\hline
\end{tabular}

Fig. 5 depicts the saturation throughput results obtained by both the analytical model and the respective simulation tool, for different optical capacity availability factors denoted by the ratio $\lambda / R$ and ranging from $10 \%$ to $90 \%$. Throughput is displayed both in its normalized form as well as in bits per second, taking into account that the maximum possible throughput value corresponds to the case where all employed RAUs can simultaneously transmit data packets at line rate.

Fig. 5(a) displays throughput for $155 \mathrm{Mbps}$ bit rate that has been already shown experimentally to allow for successful multiuser connectivity in $60 \mathrm{GHz}$ RoF networks [25]. As can be noted, throughput increases almost linearly and in total accordance with the $\lambda / R$ ratio growth. In addition, the theoretically obtained results practically coincide with the simulation-based outcomes experiencing only negligible differences up to a maximum of $1 \%$. The good agreement between theory and simulation confirms the validity of our model, revealing also the almost linear dependence between throughput and $\lambda / R$, which indicates the nearly optimum capacity exploitation offered by the proposed MT-MAC in this dual medium platform. Fig. 5(b) displays the corresponding results for the same configuration but at $1 \mathrm{Gbps}$ wireless data rate. As can be noted, the transition to a higher bit rate yields a slightly deteriorated throughput performance of up to $2 \%$ when all other network and traffic parameters remain unchanged. This owes mainly to the combined effect of the reduced packet durations obtained as the bit rate increases and of the fiber propagation delay that remains constant. The RRF duration, as defined in (13), becomes almost independent of the bit rate since the transmission delays of the relatively small-size POLL, ID, and ACK packets are negligible with respect to the fiber propagation delay $\delta_{\text {FIBER }}$. This means that the total time required for transmitting all RRFs and completing the second contention period will be almost constant and bit-rate independent, primarily determined by $\delta_{\text {FIBER }}$ since $\delta_{\text {AIR }}$ corresponds only to small cell radii. However, this will not be the case for the DFs that have a greater size than the RRFs so that their duration will still depend upon the bit rate, as can be seen in (14). Having the RRF duration constant and downlimited by the

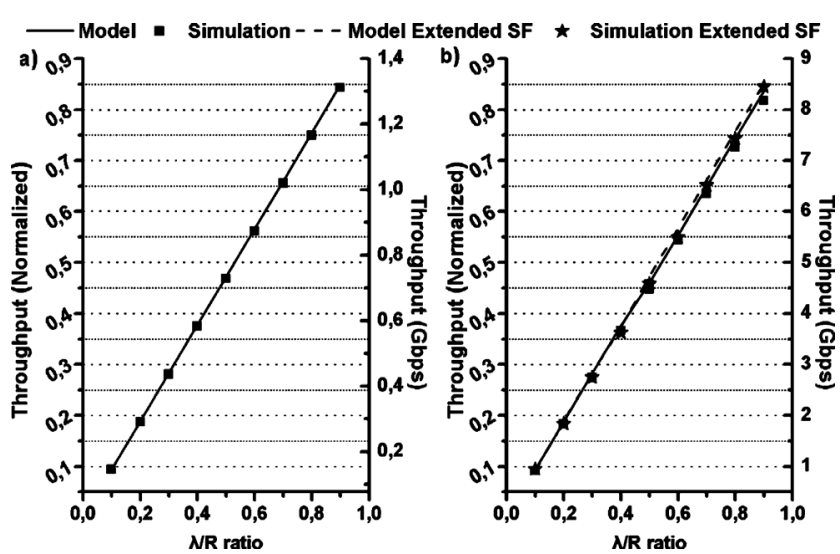

Fig. 5. (a) Throughput versus $\lambda / \mathrm{R}$ ratio performance results for wireless bit rate of 155 Mbps. (b) Throughput versus $\lambda / \mathrm{R}$ ratio performance results for wireless bit rate of $1 \mathrm{Gbps}$ with standard and extended SF size.

$\delta_{\text {FIBER }}$ quantity and the DF duration decreasing as the bit rate increases to $1 \mathrm{Gbps}$, the ratio of DFs duration within a certain SF will decrease leading to slightly lower throughput values. This can be, however, compensated by increasing the number of DFs incorporated in an SF and this case is also depicted in Fig. 5(b). The parallel extension of the $\mathrm{SF}$ to a size analogous to the bit rate increase (i.e., from 500 to 3200 for the 155 Mbps to 1 Gbps transition) renders the RRF-induced delays again negligible, owing to the enhanced number of DFs being transmitted without rerunning the second contention period. In this way, throughput performance recovers to its original metrics.

Fig. 6(a) and (b) illustrates protocol's scalability performance for a growing number of nodes per RAU and a rising number of participating RAUs in the network, respectively. Each curve represents one of three distinct $\lambda / R$ ratio configurations that were investigated, namely $30 \%, 50 \%$, and $80 \%$. Fig. 6(a) elaborates that throughput values remain practically immutable as the number of nodes grows from 2 to 20, displaying only a slight performance degradation of less than $1 \%$, mainly due to the enhanced wireless arbitration processes. The same applies to Fig. 6(b) that depicts the throughput performance as the number of RAUs employed in the network increases from 10 up to 128 , thus illustrating protocol's behavior as the optical round-trip delay multiplies, affecting in principle the RAUs located the farthest from the CO. The RAU modules are considered equally spaced with $50 \mathrm{~m}$ inter-RAU fiber intervals, producing a total fiber length between 0.5 and $6.4 \mathrm{~km}$ depending on the number of RAUs involved. As can be noted, only a small performance degradation of up to $0.5 \%$ appears in the simulation-based curve for a number of RAUs equal to 128 , while the respective curve originating from the mathematical analysis remains constant irrespective of the antennae elements incorporated. This small difference between analysis and simulation owes mainly to the additional fiber propagation delay emerging due to the considerably longer bus lengths as the number of RAUs increases, which is not taken into account in our mathematical model. It is important to note that throughput deliverance remains essentially unscathed even when the number of RAUs escalates by a factor of $1000 \%$, confirming the increased scalability characteristics 

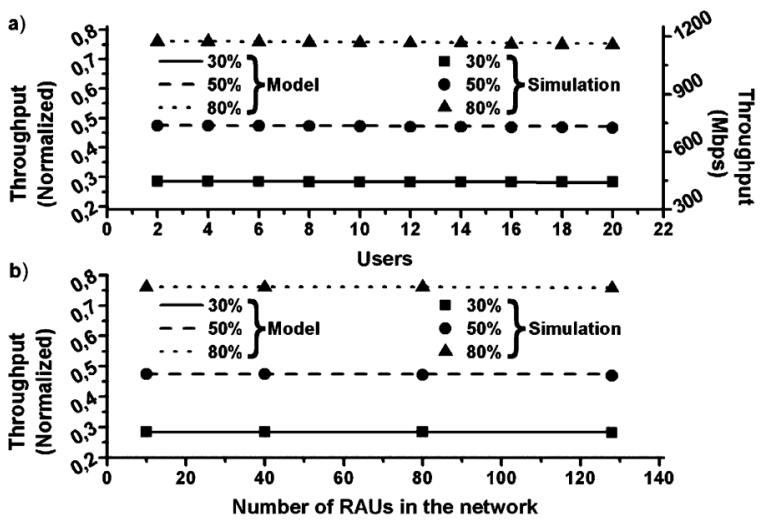

Fig. 6. (a)Throughput versus number of users per RAU for $30 \%, 50 \%$, and $80 \% \lambda / R$ ratio, respectively. (b) Throughput versus number of RAUs in the network for $30 \%, 50 \%$, and $80 \% \lambda / R$ ratio, respectively.

of the protocol and the optimum exploitation of the underlying infrastructure properties.

The validity of our model allows for its use toward acquiring a more detailed insight into the role of specific MT-MAC protocol parameters and their performance impact. Fig. 7(a) illustrates the effectiveness of the random slot number choice scheme employed in the second contention period; the probability of achieving "resolve" for ranging RRF sequence lengths is computed, assuming ten slots per RRF and different amount of competing nodes scaling from 3 to 15 (i.e., from $30 \%$ up to $150 \%$ node/slot ratios). As can be noted, "resolve" probability tends to be 1 at an early stage, essentially meaning that all wireless nodes are effectively resolved within only a handful of RRFs. The same is true even in the case where the number of nodes greatly exceeds the number of available slots, thus profitably restricting the second contention period's duration in the scope of single-digit amount of RRFs, for the benefit of throughput-producing data exchange. Based on the aforementioned, we have established the $50 \%$ users/slots ratio as a close to optimal choice for conducting the performance evaluation.

Fig. 7(b) presents both simulation and analytical throughput results for three different $\lambda / R$ ratios, namely $30 \%, 50 \%$, and $80 \%$, when a plethora of SF durations $i$ ranging from 10 to 1000 are employed and a static number of 5 wireless nodes served by each RAU at $155 \mathrm{Mbps}$ bit rate is considered. As expected, throughput performance increases with $i$, since the fraction of the second contention period duration becomes relatively insignificant as $i$ rises, so that a greater part of the SF is devoted to the DATA_TX mode and as such to actual data transmission. This can be easily explained by taking into account the fact that the quantity of RRFs required for resolving all wireless nodes is in fact independent of $i$ and as proved earlier remains minimal. Moreover, throughput tends to reach a saturation point after a certain number of frames, rendering a negligible gain in performance for greater SF sizes. This can be verified by its mere increment of only $0.3 \%$ for a frame number enhancement from 500 to 1000 , allowing us to establish the number of $i=500$ frames as a close to optimal SF size with respect to highest throughput performance. Fig. 7(c) illustrates the same evaluation for $1 \mathrm{Gbps}$ wireless bit rate. As can be observed, throughput follows the same behavioral pattern, with the only difference
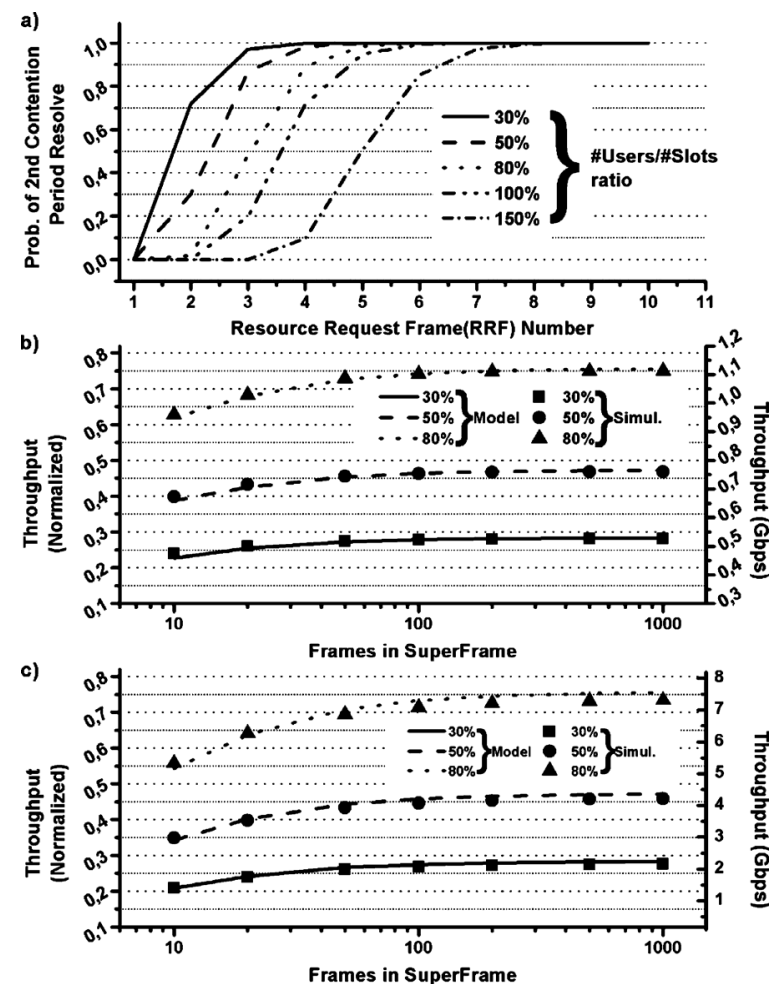

Fig. 7. (a) Analytical probability results of resolve versus number of RRFs for different users/slots ratios. (b) Throughput versus number of frames in an SF, for different $\lambda / R$ ratios at $155 \mathrm{Mbps}$ wireless bit rate. (c) Throughput versus number of frames in an SF, for different $\lambda / R$ ratios at $1 \mathrm{Gbps}$ wireless bit rate.

being that performance gains saturate at a slightly lower rate, which allows for further throughput yields as $i$ increases. The latter confirms the extended SF metrics presented in Fig. 5(b) where the enhanced DF number compensates throughput losses stemming from the manifold bit rate increase as has been analyzed earlier in this section. Finally, it can be noted that the analytical results are once again in excellent agreement with the respective simulation-based findings.

\section{DISCUSSION}

The successful MT-MAC protocol modeling provides a number of significant advantages toward turning MT-MAC schemes into reliable and viable approaches for high-bandwidth $60 \mathrm{GHz}$ wireless over fiber network applications. It confirms protocol's capability of almost optimally handling capacity offered by the two different media, extracting at the same time the optimal network and traffic parameterization conditions. Even more important, it draws the roadmap for effectively merging optical and wireless capacity arbitration algorithms within a single mathematical framework, offering the possibility to alter the complete protocol performance by modifying only the optical or only the wireless arbitration process. By combining this with the centralized topology of the proposed $60 \mathrm{GHz}$ RoF network that consolidates the complete knowledge of all network and end-user parameters into the $\mathrm{CO}$, one can reach highly agile network configurations without requiring any intervention to the hardware infrastructure.

The enhanced agility unleashed by our analytic MT-MAC model can be highlighted in a simple example of different 
bandwidth sharing strategies. The scheme demonstrated so far relies on the round-robin allocation algorithm in the optical domain, issuing a fairness policy that works on a per RAU basis so that each RAU element enjoys the same amount of throughput. However, this can produce important variations on the per node throughput values in case the distribution of wireless nodes among the RAUs experiences severe inequalities. Our analytical model can easily reveal how the fairness policy could be shifted with minor modifications to a user-centric mode of operation, where not every RAU but every single user within the entire network should perceive the same level of bandwidth. This kind of policy would demand the transition to an optical arbitration algorithm that allocates wavelengths for time windows directly proportional to the number of active nodes currently residing in each RAU. Our model shows that this can be simply realized by merely changing the optical waiting state from a "wavelength to RAU" to a "nodes to total number of nodes" ratio, without requiring any change in the wireless arbitration algorithm. This approach takes certainly advantage of the knowledge about the nodes-per-RAU distribution that can be indeed available at the $\mathrm{CO}$, since the $\mathrm{CO}$ collects all traffic requests within the RRF frames of the second contention period.

Within the same frame, the same principle could be applied solely in the wireless portion of the network as well as toward supporting a more guaranteed service in modern high-bandwidth applications with stringent delay requirements, like highdefinition video streaming. In that case, the time bandwidth allocation in the wireless arbitration process would not be distributed in equal time portions amongst active nodes, but could be in principle reserved according to specific prioritization criteria depending on the type of traffic requested. As such, requests for real time data would be ranked as higher priority traffic in the polling sequence so as to allocate continuous fractions of bandwidth. This indicates that the MT-MAC protocol can incorporate a plethora of quality of service schemes following established techniques used in a variety of wireless protocols, with the most predominant being the IEEE 802.11e standard [26].

Finally, the centralized knowledge of the entire network configuration can spur new energy reduction concepts for given 60 $\mathrm{GHz}$ network performance metrics. As the number of end users and traffic requests changes, the $\mathrm{CO}$ can update its parameter database and determine the minimum number of wavelengths required for sustaining the same level of performance. To this end, it can decide to switch off certain transceiver elements at the $\mathrm{CO}$, enabling constant and high-quality performance while preserving always the minimum possible power consumption levels.

\section{CONCLUSION}

We have demonstrated an analytical model for the MT-MAC protocol that has been recently introduced in [23] and provides dynamic allocation for both the optical as well as the wireless resources in $60 \mathrm{GHz}$ RoF networks. The analytical MT-MAC model is accompanied by a detailed saturation throughput performance analysis, assuming ideal channel conditions. Our model relies on a 2-D Markov chain approach for calculating the end-user transmission probabilities, taking into account contention for both the optical and the wireless layer resources. An analytic formula for throughput computation is derived and the respective results for different optical resource availability factors are found to be in close agreement with simulation-based outcomes, confirming the validity of the MT-MAC model. This first successful MT-MAC modeling approach indicates that the functional convergence of the optical and the wireless connection links can yield new and efficient capacity and resource utilization concepts in $60 \mathrm{GHz}$ RoF networks, equipping 60 $\mathrm{GHz}$ high-speed applications with high-level agile operational frameworks.

\section{REFERENCES}

[1] G. M. A. Ali, G. Ellinas, H. Erkan, A. Hadjiantonis, and R. Dorsinville, "On the vision of complete fixed-mobile convergence," J. Lightw. Technol., vol. 28, no. 16, pp. 2343-2357, Aug. 2010.

[2] N. Ghazisaidi, M. Maier, and C. Assi, "Fiber-wireless (FiWi) access networks: A survey," IEEE Commun. Mag., vol. 47, no. 2, pp. 160-167, Feb. 2009.

[3] M. Bakaul, A. Nirmalathas, C. Lim, D. Novak, and R. B. Waterhouse, "Simultaneous multiplexing and demultiplexing of wavelength-interleaved channels in DWDM millimeter-wave fiber-radio networks," $J$ Lightw. Technol., vol. 24, no. 9, pp. 3341-3352, Sep. 2006.

[4] C.-S. Choi, Y. Shoji, and H. Ogawa, "Millimeter-wave fiber-fed wireless access systems based on dense wavelength-division-multiplexing networks," IEEE Trans. Microw. Theory Tech., vol. 56, no. 1, pp. 232-241, Jan. 2008.

[5] M. G. Larrodé and A. M. J. Koonen, “All-fiber full-duplex multimode wavelength-division-multiplexing network for radio-over-multimodefiber distribution of broadband wireless services," IEEE Trans. Microw. Theory Tech., vol. 56, no. 1, pp. 248-255, Jan. 2008.

[6] Z. Jia, J. Yu, A. Chowdhury, G. Ellinas, and G.-K. Chang, "Simultaneous generation of independent wired and wireless services using a single modulator in millimeter-wave-band radio-over-fiber systems," IEEE Photon. Technol. Lett., vol. 19, no. 20, pp. 1691-1693, Oct. 2007.

[7] J. J. V. Olmos, T. Kuri, and K. Kitayama, "Dynamic reconfigurable WDM 60-GHz millimeter-waveband radio-over-fiber access network: Architectural considerations and experiment," J. Lightw. Technol., vol. 25, no. 11, pp. 3374-3380, Nov. 2007.

[8] L. Chen, J. G. Yu, S. Wen, J. Lu, Z. Dong, M. Huang, and G. K. Chang, "A novel scheme for seamless integration of ROF with centralized lightwave OFDM-WDM-PON system," J. Lightw. Technol., vol. 27, no. 14, pp. 2786-2791, Jul. 2009.

[9] N. Pleros, K. Vyrsokinos, K. Tsagkaris, and N. D. Tselikas, “A 60 $\mathrm{GHz}$ radio-over-fiber network architecture for seamless communication with high mobility," J. Lightw. Technol., vol. 27, no. 12, pp. 1957-1967, Jun. 2009.

[10] N. Pleros, K. Tsagkaris, and N. D. Tselikas, "A moving extended cell concept for seamless communication in $60 \mathrm{GHz}$ radio-over-fiber networks," IEEE Commun. Lett., vol. 12, no. 11, pp. 852-854, Nov. 2008.

[11] H. B. Kim, H. Woesner, and A. Wolisz, "A medium access control protocol for radio over fiber wireless LAN operating in the $60-\mathrm{GHz}$ band," in Proc. 5th Eur. Pers. Mobile Commun. Conf., Glasgow, U.K., Apr. 2003, pp. 204-208.

[12] M. J. Crisp, S. Li, A. Watts, R. V. Penty, and I. H. White, "Uplink and downlink coverage improvements of $802.11 \mathrm{~g}$ signals using a distributed antenna network," J. Lightw. Technol., vol. 25, no. 11, pp. 3388-3395, Nov. 2007.

[13] M. Mjeku and N. J. Gomes, "Analysis of the request to send/clear to send exchange in WLAN over fiber networks," J. Lightw. Technol., vol. 26 , no. 15 , pp. 2531-2539, Aug. 2008.

[14] B. Kalantari-Sabet, M. Mjeku, N. J. Gomes, and J. E. Mitchell, "Performance impairments in single-mode radio-over-fiber systems due to MAC constraints," J. Lightw. Technol., vol. 26, no. 15, pp. 2540-2548, Aug. 2008.

[15] C. Lim, A. Nirmalathas, M. Bakaul, P. Gamage, K.-L. Lee, Y. Yang, D Novak, and R. Waterhouse, "Fiber-wireless networks and subsystem technologies," J. Lightw. Technol., vol. 28, no. 4, pp. 390-405, Feb. 2010.

[16] A. Das, M. Mjeku, A. Nkansah, and N. J. Gomes, "Effects on IEEE 802.11 MAC throughput in wireless LAN over fiber systems," $J$. Lightw. Technol., vol. 25, no. 11, pp. 3321-3328, Nov. 2007. 
[17] , IEEE P802.11 ad [Online]. Available: http://www.ieee802.org/11/Reports/tgad_update.htm

[18] , IEEE $80 \overline{2} .15$ TG3c [Online]. Available: http://www.ieee802.org/15/ pub/TG3c.html

[19] C. W. Pyo and H. Harada, "Throughput analysis and improvements of hybrid multiple access in IEEE 802.15.3c mm-wave WPAN," J. Sel. Areas Commun., vol. 27, no. 8, pp. 1414-1424, Oct. 2009.

[20] C.-S. Sum, Z. Lan, R. Funada, J. Wang, T. Baykas, M. Rahman, and H. Harada, "Virtual time-slot allocation scheme for throughput enhancement in a millimeter-wave multi-Gbps WPAN system," J. Sel. Areas Commun., vol. 27, no. 8, pp. 1379-1389, Oct. 2009.

[21] WirelessHD [Online]. Available: http://www.wirelesshd.org/

[22] Wireless Gigabit Alliance [Online]. Available: http://wirelessgigabitalliance.org/

[23] G. Kalfas and N. Pleros, "An agile and medium-transparent MAC protocol for $60 \mathrm{GHz}$ radio-over-fiber local access networks," J. Lightw. Technol., vol. 28, no. 16, pp. 2315-2326, Aug. 15, 2010
[24] L. F. W. van Hoesel and P. J. M. Havinga, "Analysis of a Self-organizing Algorithm for Time Slot Selection in Schedule-Based Medium Access," Centre for Telematics and Information Technology, University of Twente, Internal Rep. TR-CTI, so:65358, 2009 [Online]. Available: http://doc.utwente.nl/65358/

[25] S. T. Choi, K. S. Yang, S. Nishi, S. Shimizu, K. Tokuda, and Y. H. Kim, "A 60-GHz point-to-multipoint millimeter-wave fiber-radio communication system," IEEE Trans. Microw. Theory Tech., vol. 54, no. 5, pp. 1953-1960, May 2006.

[26] , IEEE P802.11e [Online]. Available: http://www.ieee802.org/11/Reports/tge_update.htm

Author biographies not included at author request due to space constraints. 